Meta

Journal des tradlucteurs

Translators' Journal

\title{
BALLARD, Michel, De Cicéron à Benjamin. Traducteurs, traductions, réflexions, Lille, Presses Universitaires de Lille, 1992
}

\section{Francine Lichtert}

Volume 38, numéro 3, septembre 1993

URI : https://id.erudit.org/iderudit/003509ar

DOI : https://doi.org/10.7202/003509ar

Aller au sommaire du numéro

Éditeur(s)

Les Presses de l'Université de Montréal

ISSN

0026-0452 (imprimé)

1492-1421 (numérique)

Découvrir la revue

Citer cet article

Lichtert, F. (1993). BALLARD, Michel, De Cicéron à Benjamin. Traducteurs, traductions, réflexions, Lille, Presses Universitaires de Lille, 1992. Meta, 38(3), 568-574. https://doi.org/10.7202/003509ar

Ce document est protégé par la loi sur le droit d'auteur. L'utilisation des services d’Érudit (y compris la reproduction) est assujettie à sa politique d'utilisation que vous pouvez consulter en ligne.

https://apropos.erudit.org/fr/usagers/politique-dutilisation/
Cet article est diffusé et préservé par Érudit.

Érudit est un consortium interuniversitaire sans but lucratif composé de l’Université de Montréal, l'Université Laval et l'Université du Québec à Montréal. Il a pour mission la promotion et la valorisation de la recherche. https://www.erudit.org/fr/ 
BALLARD, Michel, De Cicéron à Benjamin. Traducteurs, traductions, réflexions, Lille, Presses Universitaires de Lille, 1992.

Dans la foulée du regain d'intérêt que la traduction connaît aujourd'hui, Michel Ballard nous propose une approche historique, tant de la pratique de la traduction que de la réflexion qu'elle a suscitée. On mesure à peine l'importance de cette activité au cours des siècles; il n'est donc pas surprenant que la mise au jour de l'histoire de la traduction dans son ensemble soit un processus laborieux et fragmenté auquel des chercheurs de toutes appartenances apportent leurs contributions, chacun en fonction de ses intérêts propres. C'est pourquoi les enseignants, les étudiants ou les professionnels qui s'interrogent, réservent généralement un accueil favorable aux tentatives de regrouper dans le cadre d'un schéma plus général les diverses considérations historiques sur la traduction. L'étude de Ballard, comme son titre l'indique, en est une d'envergure; son propos est de couvrir la période qui va de Cicéron à Benjamin. L'auteur entend «non seulement [...] examiner la traduction comme pratique, telle qu'elle s'est développée et a été perçue ou décrite, mais aussi [...] prendre en compte ce qui s'est développé comme théorisation ou comme réflexion à partir de, ou tout au moins en liaison avec cette pratique» (p. 13). Une telle mise en perspective est toujours salutaire, ne fût-ce que pour rappeler qu'en traduction, il y a toujours plus de protagonistes qu'il n'y paraît au premier coup d'œil.

Ballard adopte l'ordre chronologique pour répartir le contenu de son exposé sur cinq chapitres qui correspondent plus ou moins aux périodes suivantes: Antiquité, Moyen Âge, Renaissance, $\mathrm{XVII}^{\mathrm{e}}$, et du XVIII ${ }^{\mathrm{e}}$ siècle à la période contemporaine. Cette division traditionnelle a l'avantage de mettre d'accord les traductologues qui ne s'entendent pas nécessairement toujours sur la délimitation des étapes importantes marquant l'histoire de la traduction.

La première représentation officielle de la fonction d'interprète apparaît sur les tombes des princes d'Eléphantine (troisième millénaire avant notre ère). Michel Ballard s'en sert comme point de départ pour un survol rapide de la période antique - l'Égypte, Sumer, la Grèce et Rome - et nous mène de Platon et Aristote à Philon, saint Jérôme et saint Augustin. Avec ce premier chapitre qui s'intitule «Aux sources d'une opposition fondamentale ${ }^{1}$ », l'auteur marque déjà les points d'ancrage qui lui permettront de tisser 
des liens entre les différentes époques. C'est l'un des grands mérites du livre que d'avoir mis en lumière à l'aide de rapprochements pertinents ce réseau de fils conducteurs qui relient entre eux des traducteurs d'horizons ou de périodes différentes, la chaîne maîtresse dans ce domaine étant bien entendu la Bible. Michel Ballard nous montre ainsi où et quand les grandes idées prennent leurs sources et comment elles se frayent un chemin à travers le paysage historique. Citons à titre d'exemples la traduction comme révélation (p. 33 ), la tradition herméneutique (p. 35), l'idée des langues inférieures qui porte en elle le germe du caractère dégradant de la traduction (pp. 35-36, 86), le caractère transgressant de celle-ci (p. 38). Au chapitre des tendances générales mises en lumière tout au long de cette étude, il y a notamment la place de choix occupée par les Anciens qu'il faut imiter ou par rapport à qui il faut se justifier (p. 55), le rôle de frein de l'Église qui ira jusqu'à obliger saint Jérôme à «paraître raisonner mal» (p. 55). La métaphore de la traduction comme «lutte» qui apparaît avec ce dernier est ainsi rapprochée de la notion de la traduction dynamique de Nida ou de Cary (p. 49).

Le deuxième chapitre commence avec le philosophe Boèce (480-524) qui, luimême traducteur du néo-platonicien Porphyre (234-305), exige, par souci de vérité, le mot à $\operatorname{mot}^{2}$. Cette question de la littéralité se retrouve tout au long du Moyen Âge, quelles que soient les raisons pour lesquelles on traduit: en Europe orientale et dans le monde anglo-saxon pour évangéliser - Ballard voit en Aelfric un précurseur de Luther (p. 63) - en Orient, pour transmettre un héritage culturel, dans le sud de l'Italie et en Espagne pour assurer la diffusion, par le latin et le vernaculaire, des manuscrits grecs et arabes. En France, apparaît dès la fin du Moyen Âge, avec le souci de faire connaître à un public plus large des textes jusque-là réservés aux clercs et aux lettrés, celui de la clarté et de l'élégance. Ce chapitre est extrêmement riche du point de vue informatif (les sections consacrées à Bagdad, à Tolède et au réseau de traducteurs «mobiles» du XII ${ }^{\mathrm{e}}$ siècle sont particulièrement bien documentées). L'auteur y traite notamment de l'influence des princes commanditaires sur le contenu des préfaces et sur la manière de traduire. Par ailleurs, il attribue le souci d' «embellir pour compenser» à la prise de conscience du caractère inférieur des langues vulgaires. «Ce souci [...], écrit-il, est le second point de contact que la traduction entretiendra avec la rhétorique. Le premier ayant été celui établi par Cicéron sous la forme de l'imitation.» (p. 87). Ballard rappelle également que si les premiers érudits italiens tels que Pétrarque et Boccace furent les précurseurs de l'humanisme, ils ne touchaient qu'un public étroit (p. 89). Pour lui, «Ce sont les traducteurs qui vont ultérieurement contribuer à diffuser le goût des lectures antiques auprès d'un public plus large.» À la lecture de ce chapitre, on regrette cependant que les passages d'une région - ou d'une époque à l'autre - ne sont pas clairement marqués dans le texte. Cette remarque vaut d'ailleurs pour l'ouvrage dans son ensemble.

La Renaissance se caractérise par une intensification de l'activité de traduction que l'auteur rapproche de celle qui a suivi la seconde guerre mondiale. En titrant ce troisième chapitre «La traduction comme découverte et comme horizon», Ballard résume parfaitement l'importance de ce phénomène. Cette découverte de la culture antique, c'est assurément la traduction qui l'a rendue possible. C'est elle aussi qui, à la Renaissance, est, selon les mots d'Antoine Berman ${ }^{3}$ cités par Ballard, «l'horizon de toute écriture [...] [,] la matrice de ce que l'on commence justement à appeler licterature». Après avoir donné un aperçu des principales causes de cet accroissement - chute de 1'Empire romain d'Orient, humanisme, redécouverte de l'Antiquité, amélioration des procédés de fabrication des livres, Réforme - l'auteur rappelle le rôle que jouèrent les érudits byzantins émigrés après la prise de Constantinople dans l'enseignement du grec, de la philosophie et des lettres. Il passe ainsi en revue les principaux acteurs de la Renaissance italienne en signalant leurs contributions marquantes. Outre le fait que ces lettrés sont les auteurs de nombreuses 
traductions des philosophes grecs, on constate que des réfugiés tels que Théodore Gaza (1398-1475) et Jean Laskaris (1445-1534) ont chacun rédigé une grammaire grecque, que le chancelier de la république florentine Léonardo Bruni (1370-1444) a traduit lui-même Platon et Aristote et qu'il est l'auteur d'un traité dans lequel il insiste sur la nécessité de bien connaître les deux langues de travail - «exigence qui sera reprise par Dolet», rappelle Ballard. C'est Cosme de Médicis (1389-1464), fondateur de la première bibliothèque publique, qui encouragea Marcile Ficin (1433-1499) à traduire Platon et les platoniciens. Ballard, reprenant les chiffres de Paul Herbert Larwill ${ }^{4}$, nous signale qu'au début du $\mathrm{XVI}^{\mathrm{e}}$ siècle, «la proportion des publications en langue vulgaire par rapport à celles en latin passe de $1 / 30$ à $1 / 3$ à Venise et $3 / 1$ à Florence» alors que jusque-là les traductions se faisaient surtout du grec au latin.

Ce mouvement n'a pas la même ampleur en France. Des signes de réflexion sur la traduction se manifestent cependant. Ballard repère notamment l'apparition de préoccupations concernant les étoffements et retranchements. Avec Guillaume Tardiff, lecteur de Charles VIII, apparaît «l'introduction de mots nouveaux dont on donne une définition». Sous le règne de Louis XII, Claude de Seyssel traduira de façon indirecte les auteurs grecs à partir de traductions en latin. (Cette pratique se retrouvera en Angleterre, nous signale Ballard.) De Seyssel considère qu'il faut suivre de près le style du latin parce que «c'est le vray moyen de communiquer la langue latine avec la Françoise $»^{5}$ (p. 100). Dans cette phrase, l'auteur décèle un signe avant-coureur des théories allemandes de la seconde moitié du XVIII ${ }^{\mathrm{e}}$ siècle. Par ailleurs, on constate à la Renaissance qu'il y a davantage de liberté dans la traduction poétique, qui se fait le plus souvent en vers. L'usage de l'étoffement synomymique, qui se généralisera au $X^{\mathrm{e}}$ siècle, fait partie de la rhétorique du temps $^{6}$. À la fin du XVe $\mathrm{XV}^{\mathrm{e}}$ siècle, des érudits grecs, italiens et français enseignent le grec en France. Ce sont leurs élèves qui, avec le soutien du roi, des nobles et des bourgeois, et à l'encontre des positions des théologiens et de la magistrature, vont entreprendre de traduire les anciens. On constate, écrit Ballard, «une certaine continuité dans le refus du littéralisme», et plus loin: «on s'achemine vers un style de traduction qui culminera avec Amyot et qui parfois même annonce les libertés que Perrot d'Ablancourt prendra avec le texte pour le rendre accessible». L'auteur se demande si la dichotomie entre la Pléiade et l'école marotique est aussi nette que l'écrit Horguelin ${ }^{7}$, tout en reconnaissant que le manifeste de Du Bellay marque une étape importante. Ce n'est malheureusement que plusieurs pages plus loin (p. 122) qu'il précisera son propos en signalant que Du Bellay oppose en quelque sorte un inutile irréalisable (traduction des poètes et orateurs) d'un côté à un utile réalisable de 1'autre (traduction scientifique). C'est cette opposition qui explique sans doute, écrit Ballard, «la préférence de certains 'traducteurs' comme d'Ablancourt pour 'l'imitation' à la fois pour se libérer des critiques des littéralistes et du mépris des créateurs» (p. 122). À propos de Sébillet, et reprenant chez Horguelin une citation dans laquelle Sébillet enjoint d'imiter Marot «en ses Psalmes», il remarque que «lorsque Sébillet écrit cette louange [en 1548], Marot est mort (en exil), et que sa traduction des Psaumes n'a été appréciée que des protestants». Il faut cependant se souvenir qu'à cette époque ces derniers, fort nombreux ${ }^{8}$ et relativement plus instruits que le peuple resté catholique, représentaient une proportion non négligeable du public des lecteurs. Ballard consacre ensuite plus de 12 pages à Étienne Dolet dont il relate la vie en décrivant le contexte religieux et social de l'époque. Énonçant les cinq principes de traduction énoncés par Dolet dans son ouvrage La manière de bien traduire d'une langue en aultre: davantage, de la Punctuation de la Langue Françoise: plus des accents d'ycelle, Ballard remarque que celui-ci concrétise «une prise de conscience plus synthétique des problèmes de la traduction que les simples préfaces qui l'ont précédé» (p. 111). Il tempère pourtant cette affirmation en critiquant Cary qui, à son avis, manifeste pour le traducteur un enthousiasme dû à sa 
position de professionnel «réticent vis-à-vis de toute formalisation excessive et en particulier de l'approche linguistique». Ballard rapproche d'ailleurs le premier principe de Dolet, la compréhension du sens et de la matière qu'il traduit, de l'École de Séleskovitch et Lederer. Comparant Dolet théoricien à Luther, il lui reconnaît l'avantage d'être «laïque et universel». Même Nida, souligne-t-il, reconnaitra l'importance des principes de traduction de Dolet. Ballard remarque également que, si au milieu du XVI e siècle on assiste à la naissance des vocables «traduire», «traducteur» et «traduction», cette prise de conscience de soi correspond paradoxalement à la naissance d'un «sentiment d'insatisfaction» que l'on commence à déceler dans les préfaces. Dans les six pages qu'il consacre ensuite à l'Angleterre, l'auteur s'étendra au-delà de la Renaissance jusqu'au XVII ${ }^{\mathrm{e}}$ siècle avec le littéralisme de Ben Jonson et l'«empathie artistique» de Chapman traduisant les poètes. Suivent ensuite une quinzaine de pages consacrées à un panorama de la traduction des textes sacrés et de ses liens avec la Réforme. On reste surpris qu'à aucun moment il ne soit question de l'apport de Calvin.

Le quatrième chapitre s'intitule «Les «belles infidèles» et la naissance de la traductologie». Ballard commence par passer en revue le point de vue de quelques grands théoriciens sur le premier de ces deux phénomènes: Mounin, Cary et Zuber ${ }^{9}$ dont l'ouvrage, juge-t-il, «[contribue] à entretenir une vue partiale des problèmes de la traduction au XVII ${ }^{\mathrm{e}}$ siècle et [...] [comporte] une analyse insuffisante des textes théoriques». En effet, après avoir souligné pendant une dizaine de pages l'apport de Malherbe sur le plan de la formalisation de la langue ainsi que son influence sur la traduction, Ballard tient à rappeler que, parallèlement au mouvement qui s'écarte de l'original dans un souci de plaire, existe aussi la tendance inverse, notamment avec Pierre-Daniel Huet, les jansénistes de PortRoyal et l'académicien de Méziriac. À ce dernier, il accorde d'ailleurs une importance considérable en raison de son discours intitulé «De la Traduction», présenté à l'Académie française. Dans ce texte, qui remet en question la manière de traduire d'Amyot, Ballard voit «un travail scientifique rigoureux, l'une des premières analyses d'erreur systématiquement présentées» (p. 165) et «un des textes fondateurs de la traductologie» (p. 170). Ce texte, écrit-il, «pose les éléments d'une déontologie de l'activité traduisante tant sur le plan linguistique qu'extralinguistique». L'auteur accuse d'ailleurs tant Edmond Cary (p. 161) que Roger Zuber (p. 163) de condescendance à l'égard de l'académicien dont il reprend des passages entiers sur plusieurs pages. Il semble même que, dans cet ouvrage, Ballard se soit engagé à tirer d'un oubli relatif tous les efforts de systématisation théorique de la traduction. (On observe le même phénomène au dernier chapitre en ce qui concerne Tytler.) Ainsi, un peu plus loin (p. 176), il s'en prend à nouveau à Zuber, l'accusant d'avoir, comme d'autres d'ailleurs, sous-estimé les efforts en ce sens des pédagogues de Port-Royal (dont il traitera sur trois pages), de Pierre-Daniel Huet et de Gaspard de Tende. Il consacre d'ailleurs repectivement environ sept, deux et dix pages à ces derniers. En accordant tant d'importance à ces voix discordantes, Ballard a surtout voulu montrer que «les deux manières opposées de traduire continuent de coexister» (p. 197).

Le chapitre V s'intitule «Divergences et réactions». «Divergences» s'applique à l'Angleterre du XVII' siècle qui, après une courte période dans la tradition du littéralisme de Ben Jonson, va s'en éloigner. «Réactions» caractérise l'attitude de l'Allemagne vis-àvis de l'influence française.

En ce qui concerne l'Angleterre, Ballard passe en revue les traducteurs néoclassiques, dont les principaux représentants sont sir John Denham et Abraham Cowley. Ceux-ci, tous royalistes, ont passé plusieurs années d'exil en France, ce qui fut sans doute pour eux l'occasion de subir l'influence des idées de d'Ablancourt. Ils s'orientent donc vers une «recréation au nom de la différence culturelle et linguistique séparant le public de l'original et le public récepteur de la 'traduction'». En poésie, les idées de Chapman 
continuent à dominer et on s'écarte cette fois absolument de la langue de départ. À la fin du siècle, Dryden reprendra les mêmes idées tout en posant certaines limites à la latitude autorisée. Ballard reprend la remarque de Steiner pour qui la traduction anglaise serait davantage centrée sur le poète alors que son homologue française le serait sur le public. Il émet pourtant des réserves: le tempérament personnel du traducteur intervient énormément dans sa façon de traduire. On assiste vers le milieu du siècle avec les apothicaires londoniens, le plus souvent puritains, à un mouvement inverse. Leur principal représentant est Nicholas Culpeper. Ces traducteurs scientifiques sont avant tout des rationalistes qui «se [méfient] de l'imagination» et «[rejettent] toute utilisation débridée ou esthétisante du langage». Ballard remarque judicieusement que si, dans leur façon de traduire, ils ne font pas de différence entre le texte factuel et religieux, «leur sens de la communication les [empêche] d'adopter un littéralisme total». Pourtant, dans l'ensemble, les belles infidèles persisteront au cours du XVIII , bien que dès le tournant du siècle, Edward Sherburne et au début du XVIII ${ }^{\mathrm{e}}$, Alexander Pope et ensuite Alexander Fraser Tytler viendront moduler cette manière de faire. Ballard consacre plusieurs pages à Tytler (212 à 225) et passe en revue la manière dont divers auteurs ont traité de ce dernier: Larbaud, George Steiner, T.R. Steiner, Nida, Kelly. Comme précédemment pour Méziriac, Ballard vole d'ailleurs à la défense de Tytler lorsqu'il estime que T.R. Steiner lui manifeste de la condescendance. Présentant en détail l'Essay on the Principles of Translation (1790), communication présentée devant la Royal Society d'Edimbourg, il le qualifie de «travail original par son ampleur et sa systématisation» (p. 223). Il le considère même comme «aussi important que celui de Gaspard de Tende par son ampleur et son sérieux» (p. 224). Par rapport à ce dernier, Tytler va au-delà des questions linguistiques pour se préoccuper davantage du style.

C'est en Allemagne qu'apparaîtront les réactions les plus vives contre la tradition française, dont Johan Christoph Gottsched sera l'un des derniers représentants au XVIII ${ }^{\mathrm{e}}$ siècle. À peu près vers la même époque - ici Ballard cite Berman: «La théorie allemande de la traduction se construit consciemment contre les traductions à la française» (pp. 228229) —, apparaissent les pionniers de la nouvelle manière de traduire, c'est-à-dire selon André Lefevere ${ }^{10}$ : Bodmer, Breitinger, Lessing et Herder, dont les idées seront reprises par les maîtres: Goethe et Schleiermacher. L'auteur expose brièvement les idées principales de ces novateurs en insistant sur l'élargissement du concept de traduction aux problèmes du langage apporté par Herder et sur le concept de «Bildung», reprenant en cela l'essentiel des analyses d'Antoine Berman. Il décrit ensuite brièvement les trois modes de traduction présentés par Goethe dans Le Divan occidental-oriental (1819) qui représente pour Berman «l'expression la plus achevée de la pensée classique allemande sur la traduction ${ }^{1}{ }^{1}$. Le premier et le deuxième mode correspondent grosso modo l'un à la Bible de Luther, l'autre aux belles infidèles, le troisième visant à «rendre la traduction identique à l'original, en sorte qu'elle puisse valoir non à la place de l'autre, mais en son lieu»'12.

Ballard retrouve dans l'Angleterre du XIX ${ }^{\mathrm{e}}$ siècle un courant central correspondant à cette façon, préconisée par Goethe, de respecter le plus possible la forme du texte original. Dans les dix-huit pages qui suivent, il sera question de la manière de traduire des préraphaélites et de celle des victoriens, toutes deux influencées par un certain littéralisme. Sept pages traitent de la querelle qui oppose les universitaires Matthew Arnold et Francis W. Newman à propos de la traduction d'Homère. Saluant le fait qu'Arnold se pose en critique de la traduction, Ballard remarque que leur polémique porte avant tout sur la définition de la fidélité en traduction, qu'ils préconisent tous deux. Pour Arnold, être fidèle revient à s'adresser à un public d'érudits (qui est capable de lire l'original et de juger de la traduction), alors que pour Newman, c'est le grand public qu'il faut séduire. Tous deux veulent préserver l'étrangeté du texte comme le demande la tradition allemande, 
mais s'en écartent chacun à leur manière: le premier en réclamant l'utilisation (peu fréquente en anglais) de l'hexamètre tout en utilisant une langue «lisible», le second en adoptant un langage archaïque, mais une forme familière, la ballade romantique. On déplore ici que Ballard n'ait pas mis en parallèle le texte de Leconte de Lisle. Ou encore qu'à propos des traducteurs orientalistes, il ne fasse qu'une brève allusion au texte de Galland pour dire qu'il servit de base à plusieurs retraductions. De même, lorsque l'auteur rappelle que Lemaire rapproche Rosetti traduisant la poésie de Dante de Baudelaire et Mallarmé traduisant Poe, on regrette que se dessine un créneau, un vide curieux, dans ce panorama historique: l'auteur semble en effet vouloir éviter de traiter de la traduction française, bien qu'à plusieurs reprises, il fasse allusion à l'influence des théories allemandes en France au XIX ${ }^{\mathrm{e}}$ siècle ${ }^{13}$ et qu'il renvoye de manière indirecte à l'ouvrage de Lieven d'Hulst, Cent ans de théorie française de la traduction. De Batteux à Littré. Cette lacune apparaît comme une incohérence par rapport à l'ensemble de l'ouvrage.

Après cette incursion en Angleterre, Ballard revient à l'Allemagne en consacrant environ six pages à Walter Benjamin, critique, essayiste et traducteur de la première partie du XX $X^{e}$ siècle. Il analyse en profondeur son essai sur «La tâche du traducteur», publié en exergue de sa traduction des Tableaux parisiens de Baudelaire. «La traduction, écrit Ballard, [y] est envisagée à la fois comme continuation de l'œuvre (dans sa vie et sa survie) et comme relation intime entre les langues [...]» (p. 256). Et plus loin : «'objet de la traduction n'est pas le sens mais une essence spirituelle qui procède de l'identité de la langue qui l'exprime. Par la traduction et au travers de l'altérité de la langue de traduction, l'œuvre se trouve exprimée avec plus de vérité dans une tension vers le langage pur [...]». L'approche de Benjamin continue d'être reprise par des théoriciens contemporains. On songe, écrit Ballard, à Meschonnic pour les textes sacrés, Saint-John Perse en poésie ou Klossowski traduisant l'Énéide. Une fois de plus, on se prend à regretter que l'auteur n'ait pas abordé dans ce chapitre le domaine français.

En guise de conclusion, Ballard commence par récapituler le contenu de ses chapitres précédents auxquels il ajoute de brèves remarques portant sur l'époque française qu'il n'a pas couverte. Il est question par exemple de Voltaire ou de Hugo fils. Sont évoqués aussi, dans cette synthèse globale, les différentes traductions de la Bible ainsi que le phénomène de la retraduction en général pour aboutir en toute logique au caractère «non fini» de la traduction. Ce que l'auteur a voulu exprimer, c'est que le «sentiment de continuum» que l'on observe dans la réflexion linguistique au cours des âges peut être «transposé dans le domaine traductologique». Il y a fort bien réussi. Remarquant qu'il est courant en traduction de parler d'un clivage entre théoriciens et praticiens, Ballard estime qu' «au contraire, ce sont les traducteurs eux-mêmes qui éprouvent le besoin, de façon compulsive, de parler de leur travail» (pp. 273-274). Cela, ils l'ont fait de façons diverses - remarques incidentes, préfaces et traités - mais ce qui, pour Ballard, caractérise le «passage à la traductologie», c'est le fait de quitter le problème immédiat pour le replacer dans un contexte plus vaste. Il situe ainsi la véritable naissance de la traductologie avec Méziriac et Gaspard de Tende. Il estime cependant que la traductologie ne doit pas se limiter à son aspect critique, ce qui risquerait de devenir normatif. Insistant sur le rôle d'observateur du traductologue, il considère plutôt la traductologie comme une science descriptive.

C'est dans cet ordre d'idées que son ouvrage contribuera certainement, par sa richesse d'information et ses tentatives de synthèses, à l'avancement des études de traductologie. Notons encore qu'outre le foisonnement de données concernant la traduction proprement dite qu'il nous fournit, Ballard nous signale également au fil des périodes les publications importantes au point de vue lexicographique, stylistique ou grammatical. Le livre contient une bibliographie ainsi qu'un index des noms propres. Dans un ouvrage d'une telle ampleur, il est inévitable qu'apparaissent certaines lacunes. Dans ce cas-ci, 
c'est l'omission de la période française qui va du XVIIIe siècle à l'époque contemporaine qui est la plus regrettable. Ceci nous amène tout naturellement au principal défaut du livre, qui réside dans sa forme. En effet, l'ouvrage donne l'impression d'avoir été publié à la hâte. Tant la construction que la typographie laissent à désirer, ce qui rend la lecture malaisée. À l'intérieur des chapitres, les divisions et les enchaînements ne sont pas clairement marqués. La toilette du manuscrit ne semble pas avoir été faite: de nombreuses fautes subsistent. À titre d'exemples on trouve des fautes aux noms propres (Lefèvére p. 16, Valéry Larbaud, Fédorov p. 18); une ponctuation incohérente, surtout dans la manière de présenter les citations (p. 60); des coquilles (le pierre de rosette, p. 30); des fautes d'orthographe et de grammaire (c'est à dire p. 39, des feuillets constituants un codex, p. 44), etc. De telles fautes, bien qu'elles soient nombreuses, sont réparables. Il faut donc espérer que cet ouvrage, par ailleurs excellent, paraitra bientôt sous une forme révisée, car il constituera certainement un outil incontournable pour tous ceux qu'intéressent l'histoire et la théorie de la traduction.

\section{Notes}

1. Dans Traduire: Théorèmes pour la traduction (Payot, Paris, 1979), J.-R. Ladmiral a explicité très clairement comment il conçoit ce qui m'apparaît être cette opposition dans un chapitre consacré à «La problématique de l'objection préjudicielle». Il explique le fait «qu'en matière de traduction théorie et pratique aient deux histoires parallèles, en contradiction l'une avec l'autre» par l'existence d'une antinomie fondamentale : «La traduction est impossible, et/ou: Tout est traduisible [...].» Ce «problème de l'intraduisibilité [...] se répercute, au niveau de la pratique traduisante, dans les termes opposés d'une alternative, elle-même «antinomique»: faut-il traduire près du texte ou loin du texte? Traduction littérale ou traduction littéraire (dite «libre»); la fidélité ou l'élégance; la lettre ou l'esprit... Là encore, ce sont les deux pôles d'une même alternative, indéfiniment rebaptisés, qui scandent l'histoire de la traduction $[\ldots . .] .$.

Et plus loin : «[...] il faut satisfaire là simultanément à deux exigences apparemment contradictoires, et qui sont en fait les deux faces d'une seule et même, double, exigence. Il faut à la fois la fidélité et l'élégance, l'esprit et la lettre...

La théorie de la traduction, ou «traductologie» est pleine de ces couples d'opposés qui répercutent ou «reproduisent» une structure fondamentalement antinomique. Au-delà de la division du travail qu'on vient de mentionner, entre une aristocratie d'esthètes et un prolétariat de traducteurs, il s'agit en l'occurrence plus fondamentalement de la tension contradictoire qui définit le rapport entre théorie et pratique [...]» (pp. 89-90).

2. Ici, Ballard cite Louis Kelly, The True Interpreter, Oxford, Blackwell, 1979. Remarquons à cette occasion que l'auteur fait fréquemment référence à des auteurs qui, comme lui, ont contribué à rassembler des données historiques, à les commenter et à les présenter de façon synthétique. Parmi les noms qui reviennent le plus souvent figurent ceux de Susan Basnett-McGuire, Paul Horguelin, Georges Mounin et George Steiner.

3. L'épreuve de l'étranger. Culture et traduction dans l'Allemagne romantique, Paris, Gallimard, 1984.

4. La théorie de la traduction au début de la Renaissance, Münich, Wolf, 1934.

5. Ballard reprend une citation de Paul Herbert Larwill, ibid.

6. Ballard fait cette remarque en se référant à des conclusions tirées par Larwill.

7. «Deux grandes écoles s'opposent tout au cours du siècle. D'une part, l'école marotique qui considère la «version» comme un genre littéraire et un moyen de «décorer» la langue. D'autre part, le groupe de la Pléiade qui veut faire du français une langue littéraire : l'enrichir en empruntant aux Anciens, aux patois et aux langues de métier, mais aussi le défendre contre les traductions parce qu'elles remplacent la création en langue vernaculaire.» Horguelin, Anthologie de la manière de traduire. Domaine français, Linguatech, 1981, cité par Ballard p. 104.

8. Dans L'homme protestant (Hachette, 1980), Janine Garrisson-Estèbe estime qu'autour des années 1560, la population protestante représente environ $10 \%$ du royaume (p. 21$)$.

9. Roger Zuber, Perrot d'Ablancourt et ses «Belles Infidèles». Traduction et critique de Balzac à Boileau, Les Presses du Palais Royal, Paris, 1968.

10. P. 229, Ballard se réfèe à André Lefevere, Translating Literature: the German Tradition, from Luther to Rosenzweig, Assen / Amsterdam, Van Gorcum, 1977, p. 1.

11. Berman cité par Ballard, p. 223.

12. Ballard citant Goethe cité par Berman, p. 96.

13. Note 110, p. 251.

FRANCINE LICHTERT Montréal, Canada 